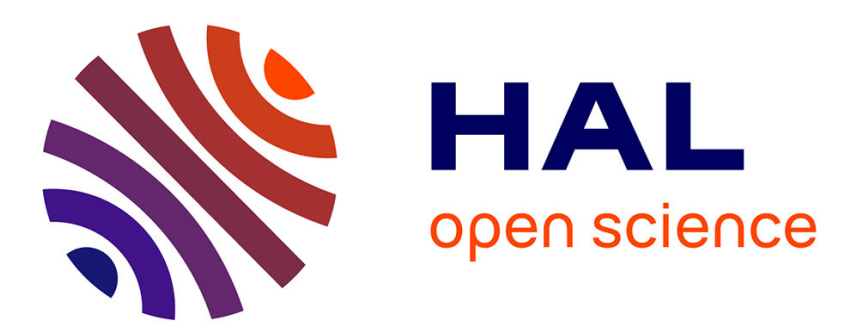

\title{
Acceleration of 3D DOSY NMR by Spatial Encoding of the Chemical Shift
}

Corentin Jacquemmoz, Jean-Nicolas Dumez

\section{To cite this version:}

Corentin Jacquemmoz, Jean-Nicolas Dumez. Acceleration of 3D DOSY NMR by Spatial Encoding of the Chemical Shift. ChemPhysChem, 2018, 19 (23), pp.3204-3210. 10.1002/cphc.201800771 . hal-02348470

\author{
HAL Id: hal-02348470 \\ https://hal.science/hal-02348470
}

Submitted on 19 Jan 2021

HAL is a multi-disciplinary open access archive for the deposit and dissemination of scientific research documents, whether they are published or not. The documents may come from teaching and research institutions in France or abroad, or from public or private research centers.
L'archive ouverte pluridisciplinaire HAL, est destinée au dépôt et à la diffusion de documents scientifiques de niveau recherche, publiés ou non, émanant des établissements d'enseignement et de recherche français ou étrangers, des laboratoires publics ou privés. 


\section{Acceleration of 3D DOSY NMR by spatial encoding of the chemical shift}

\author{
Corentin Jacquemmoz ${ }^{[a]}$ and Jean-Nicolas \\ Dumez ${ }^{[\mathrm{a}] \star}$
}

\begin{abstract}
Diffusion-ordered NMR spectroscopy (DOSY) is a powerful method for the analysis of solution mixtures. With 3D DOSY, the 2D NMR spectra of a mixture's components can be separated according to the translational diffusion coefficient of each component. The acquisition of 3D DOSY data is however very time consuming, because of the need to consecutively acquire scans for both the diffusion and the indirect spectral dimensions. We show that spatial encoding of the indirect spectral dimension, of the kind used in ultrafast 2D NMR, can accelerate 3D DOSY experiments by an order of magnitude or more. This is illustrated with homonuclear single-quantum (COSY) and double-quantum (DQS) correlation spectra. Implementations with concatenated and incorporated (iDOSY) diffusion blocks are compared and in both cases, 2D spectra are separated with less than 6 min of experiment time.
\end{abstract}

\section{Introduction}

Nuclear magnetic resonance (NMR) spectroscopy is an essential analytical technique for chemistry. NMR is used for a variety of purposes including identification and quantification, structure elucidation, kinetic measurement, titration, etc. In many cases, the sample of interest consists of a solution mixture. Within the suite of NMR experiments, one of the most relevant for mixture analysis is diffusion-ordered NMR spectroscopy (DOSY), ${ }^{[1],[2]}$ which makes it possible to separate signals and entire spectra according to the translational diffusion coefficient of the corresponding molecule.

In DOSY experiments, the diffusion information is encoded by applying a pair of field gradient pulses separated by a fixed delay. A series of spectra are acquired for increasing values of the gradient amplitude, and the diffusion-attenuated signals are fitted with the Stejskal-Tanner equation:

$$
I(g)=I_{0} e^{-D g^{2} \gamma^{2} \delta^{2} \Delta^{\prime}}
$$

where $I(g)$ and $I_{0}$ are the intensity of the signal with and without the diffusion-encoding gradients, $D$ is the diffusion coefficient of the compound, $\gamma$ the gyromagnetic ratio of nucleus used for diffusion encoding, $\Delta^{\prime}$ the delay between the encoding and decoding gradient (corrected for finite pulse width), $\delta$ and $g$ the duration and intensity of the encoding gradient. The DOSY concept is applicable to the separation of near-arbitrary $\mathrm{nD}$ spectra and requires the acquisition of $a(n+1)$-dimensional data

[a] C Jacquemmoz, Dr J-N. Dumez

Institut de Chimie des Substances Naturelles, CNRS UPR2301, Univ. Paris Sud, Université Paris Saclay, Avenue de la Terrasse, 91190 Gif-sur-Yvette, France

E-mail: jeannicolas.dumez@cnrs.fr set.

DOSY is most frequently used to separate 1D spectra, with the diffusion gradient pulses included in a spin or stimulated echo. While this method is really efficient when signals are well resolved, for overlapped signals the modelling of the diffusion curve is more complex and loses in accuracy. Multivariate processing methods exist that can address the problem of overlapped spectra. ${ }^{[3]}$ Another approach to deal with crowded spectra is to spread signals over two spectral dimensions to decrease the amount of signal overlap. This 3D DOSY approach has been demonstrated with a variety of $2 \mathrm{D}$ experiments; ${ }^{[4-8]} \mathrm{a}$ further advantage is that 3D DOSY spectra combine the separation power of DOSY with the information-content that $2 \mathrm{D}$ spectra provide, such as connectivity (COSY, HSQC...) or spatial organization (NOESY, HOESY...). The applicability of 3D DOSY is however limited by the long experiment duration required for the acquisition of multidimensional experiments with two incremented dimensions (the indirect spectral dimension and the diffusion dimension). This long duration (from $3 \mathrm{~h}$ to more than $14 \mathrm{~h}$ when phase cycling is needed) can be a problem. For samples that evolve over time (degradation, reaction monitoring...) more artefacts (such as $t_{1}$-noise) are expected to appear for long experiments. Moreover the measured diffusion coefficient will be influenced by the appearance of disappearance speed of the compounds.

Multidimensional NMR experiments can be accelerated in a number of ways, and several of them have already been exploited for 3D DOSY..$^{[9-11]}$ For example, Hadamard encoding can be used when a small number of resonances is present in the $1 \mathrm{D}$ spectrum. ${ }^{[10]}$ Sparse $^{[9]}$ and accordion ${ }^{[11]}$ samplings of the incremented dimensions have also been considered. For $2 \mathrm{D}$ NMR, the fastest methods rely on spatial encoding of the indirect dimension, as proposed by Frydman and co-workers for indirect spectral dimensions ${ }^{[12-14]}$ and Keeler and co-workers for the diffusion dimension. ${ }^{[15],[16]}$ This so-called ultrafast approach makes it possible to decrease the experimental time needed for a $2 \mathrm{D}$ acquisition from several minutes to less than one second. It has already been studied for $2 \mathrm{D}$ experiments such as COSY, ${ }^{[17]}$ J-resolved, ${ }^{[18]} D Q S^{[19],[20]}$ and also with pseudo $2 \mathrm{D}$ experiments such as DOSY[21],[22] or diffusion-relaxation correlation experiments. ${ }^{[23]}$ For 3D DOSY, either the indirect spectral or the diffusion dimension could in principle be spatially encoded, and the latter option has been explored recently. ${ }^{[22]}$

In this paper, we show that the acquisition of 3D DOSY spectra can be accelerated by one order or magnitude or more, with spatial encoding of the indirect spectral dimension. The virtual separation of homonuclear correlation spectra is demonstrated, with experiment durations of less than 6 minutes. Several implementations of the experiment are compared, and the relative merits of spatially encoding the indirect spectral and the diffusion dimension are discussed. The proposed 3D DOSY-UFNMR approach should be a practical and useful tool for the analysis of solution mixtures. 


\section{Theory}

In ultrafast 2D NMR, temporal encoding of the indirect dimension is replaced by spatial encoding. This is achieved with the joint application of a magnetic-field gradient and a chirp frequency-swept pulse, which results in a position-dependent evolution period. The detection scheme consists of a train of bipolar gradient pulses, to obtain at once the spatially encoded and the direct dimension.

In order to collect 3D DOSY data based on a given 2D pulse sequence, a pair of gradient pulses should be included to encode the diffusion dimension. For conventional experiments three approaches exist: DOSY-X, where a classic DOSY pulse sequence is followed by the chosen $2 \mathrm{D}$ pulse sequence (COSY, ${ }^{[4]} \mathrm{DQS}, \mathrm{HSQC} \ldots$ ), X-DOSY, where the 2D pulse sequence is followed by the DOSY one,$^{[8]}$ and the iDOSY approach where the DOSY pulse sequence is integrated in the 2D pulse sequence..$^{[5-7]}$ Figure 1 shows two examples of COSYDOSY pulse sequences. Any of these methods could in principle be implemented with spatial encoding of the indirect spectral dimension.

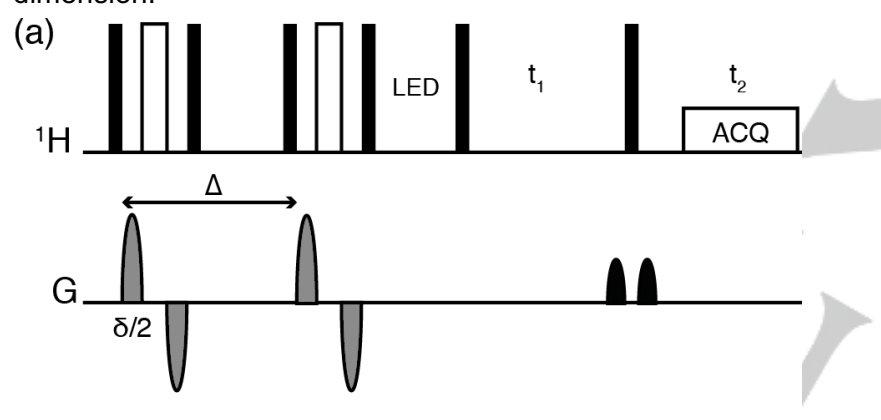

(b)

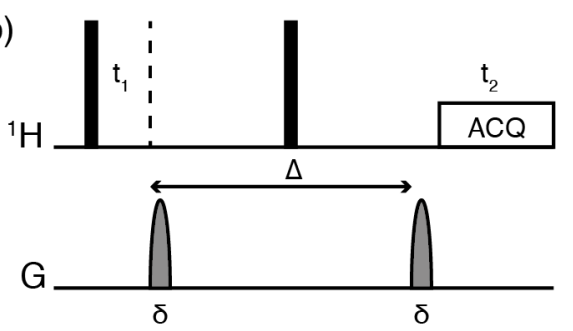

Figure 1: Pulse sequences for (a) DOSY-COSY and (b) COSY-iDOSY. For DOSY-X, the pulse sequence used for the DOSY part includes a longitudinal delay (LED) and bipolar gradient pulses. Black and white lines respectively represent $90^{\circ}$ and $180^{\circ}$ pulses. Diffusion gradients are represented in gray and coherence selection gradients in black.

Figure 2.a) shows the proposed pulse sequence for DOSYufCOSY. In this implementation, diffusion and spatial encoding are fully separated. The use of a stimulated echo, however, results in a loss of half of the signal. Moreover STE pulse sequences usually need a long phase cycling, increasing the experimental time, although this may be circumvented with the Oneshot approach ${ }^{[24]}$ or orthogonal coherence-selection gradients.
An alternative implementation of fast 3D DOSY is based on the iDOSY approach. The COSY-iDOSY sequence is appealing, as it is expected to be more sensitive and can help to observe signals for weakly coupled spins. ${ }^{[25]}$, The iDOSY method is however not suitable for every compound. Unlike in the stimulated echo approach, spins stay in the transverse plane during the entire diffusion delay; the method can thus be used as long as the $T_{2}$ of the observed compounds stays small compared to the relaxation delay $\Delta$. The pulse sequence for ufCOSY iDOSY is shown in Figure 2.b), based on the COSYiDOSY pulse sequence of Ref. ${ }^{[7]}$. This pulse sequence will however face sensitivity problems, because of the long delay between the spatial-encoding step and acquisition. To avoid unnecessary signal losses during the diffusion delay, a better pulse sequence for ufCOSY-iDOSY is proposed (Figure 2.c). Placing the first diffusion gradient before the beginning of spatial encoding makes it possible to minimise the diffusion-losses due to spatial encoding.

The sequence of events found in the ufCOSY-iDOSY pulse sequences can potentially complicate the analysis of the diffusion decay of the peak volumes. The Stejskal-Tanner equation shown in Eq. [1] is only valid if the spin phase before the first diffusion-gradient pulse does not vary along the gradient axis, and if no further gradients are applied along that axis during the diffusion delay. In the two pulse sequences proposed for ufCOSY-iDOSY, if the axis for spatial encoding gradient is the same as that for diffusion-encoding, not only are these conditions not satisfied, but the modification to the StejskalTanner equation is peak-dependent and difficult to calculate. The simplest way to circumvent this problem is to use orthogonal axes for spatial encoding and diffusion encoding, with the consequence that a triple-axis gradient probe is needed.

${ }^{1}$

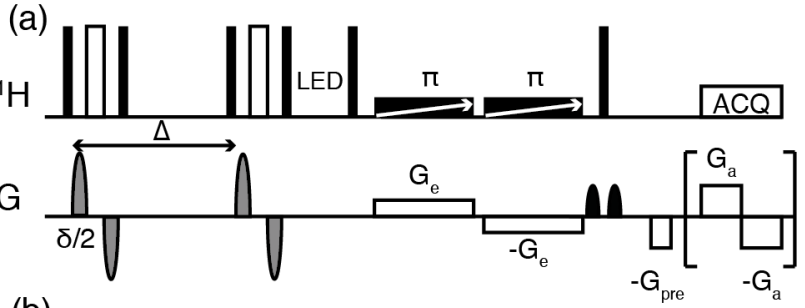

(b)
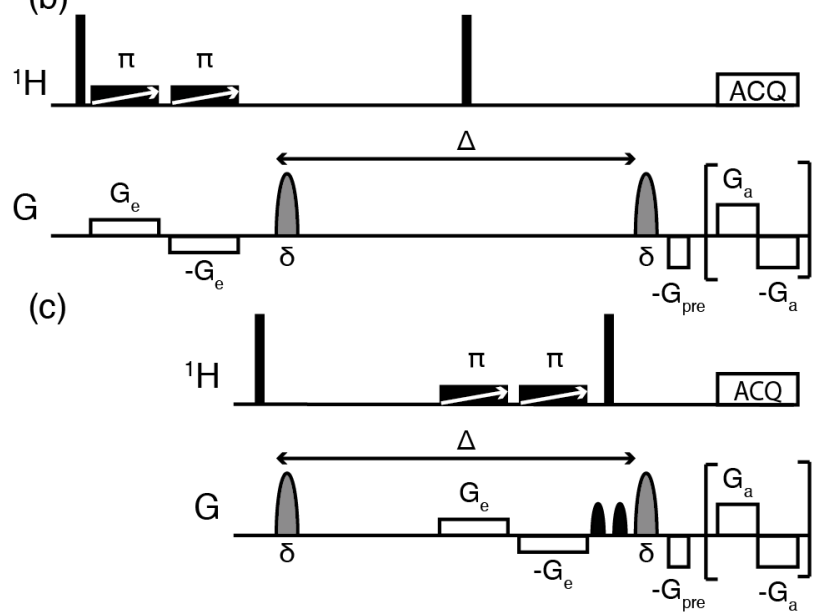
Figure 2: Pulse sequences for (a) DOSY-ufCOSY, (b) ufCOSY-iDOSY (initially proposed) and (c) ufCOSY-iDOSY (final). To give meaningful results for iDOSY, the spatial encoding and the diffusion gradient pulses must be applied on orthogonal axes. For DOSY-ufCOSY, the diffusion gradients can be on any axis, giving similar results.

Note that the ufX-DOSY has not be studied here because of the signal losses that are expected if the diffusion delay happened after spatial encoding in the pulse sequence.

\section{Results and Discussion}

\section{ufCOSY-DOSY}

3D DOSY data were collected with the DOSY-ufCOSY and ufCOSY-iDOSY pulse sequences shown in Figure 2 on a test sample containing L-valine, propanol, ethanol and methanol at a concentration of $100 \mathrm{mM}$ in $\mathrm{D}_{2} \mathrm{O}$. Figure 3.a) shows the ufCOSY spectra of the DOSY-ufCOSY data set with the weakest diffusion gradient. A good-quality spectrum is obtained and regions can be delineated for DOSY processing. Not all peaks are detected and this is expected because of the constant-time nature of the experiments. ${ }^{[25]}$ Figure 3.b) shows the evolution of the peak volumes as a function of the gradient intensity. From this figure four distinct patterns can already be seen, allowing the separation of the four compounds. Note that the residual HOD is not seen on this figure as its signal is outside the detected spectral window. (a)

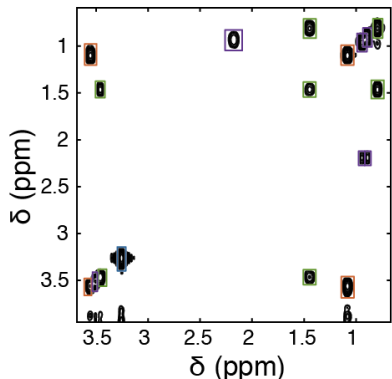

(b)

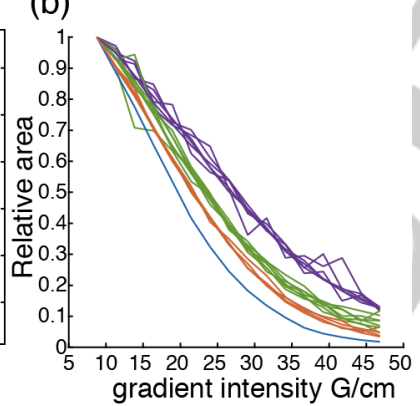

Figure 3: First slice of the DOSY-ufCOSY spectrum of a model mixture of short chain alcohols and an amino acid (corresponding to the first gradient increment) (a) and diffusion decay curves for all the peaks in the 2D COSY spectrum (b). Some peaks are missing in the spectra for valine because of $J$ modulation. The signal at the low edge of the spectrum is an artefact. Diffusion gradient were along the Z-axis.

Similar results are obtained with the ufCOSY-iDOSY pulse sequence, with the notable difference that the signal-to-noise ratio is higher since the use of a stimulated echo is avoided. Differences also arise in the relative peak intensities, as in the conventional experiments, because of J-modulation during the added delay. Spatial encoding was performed here along the Zaxis. While good results are obtained for the DOSY-ufCOSY version with diffusion gradient along $\mathrm{Z}$ or $\mathrm{X}$, diffusion-encoding along $\mathrm{X}$ was necessary to obtain meaningful results with COSYiDOSY.

Figure 4 shows slices extracted from the reconstructed 3D spectrum for the DOSY-ufCOSY data set. The spectra for all the compounds are well separated, as in the conventional DOSY COSY experiments. A notable difference is that the data shown in Figure 4 was recorded in about 3 minutes, compared to 3 hours minimum for the conventional version.

The comparison between diffusion coefficients obtained with DOSY-ufCOSY and those obtained with the conventional sequence is shown in Figure 5 (blue crosses), for experiments carried out with a classic $5 \mathrm{~mm}$ NMR tube. The mixture's components are well separated and ordered in both cases. However, a clear systematic difference is observed between the measured diffusion coefficients obtained with the two experiments. This difference is largely due to the non-uniform gradient in our triple-axis-gradient probe - the field variation when a gradient pulse is applied is larger at the centre of the RF coil than at the edges. ${ }^{[26]}$ Such gradient non-uniformity results in a subtle influence of the spatial apodisation process used in UF 2D NMR. Figure 6 shows the spatial profile obtained after 2D Fourier transformation of the rearranged UF data of the entire sample. Window multiplication by a Gaussian function is usually employed to increase signal to noise ratio and to decrease sinc wiggles in the spatially encoded spectral dimension. With nonluniform gradients, the estimated diffusion coefficient will depend on the wideness of the sample that is selected by spatial apodisation. This is illustrated in Figure 7, where the apparent diffusion coefficient is plotted as a function of the width of the window function. The measured values are found to converge for width of the window of $3 \mathrm{~mm}$ or less (the data shown in Figure 5 in blue correspond to a value of $3 \mathrm{~mm}$ for the width of the Gaussian window).

For the conventional DOSY-COSY experiment, the effect of non-uniform gradients can be reduced by using a susceptibilitymatched (Shigemi) tube with a restricted sample length. The experiment was thus repeated, with a sample length of $10 \mathrm{~mm}$, selected because further reduction of the sample length did not change significantly the measured diffusion coefficient noticeably. An updated comparison of diffusion coefficients obtained with the conventional and UF COSY DOSY sequence is shown in Figure $\mathbf{5}$ (red crosses). The systematic deviation is largely reduced. Table 1 summarises the diffusion coefficient values obtained in conventional tube, Shigemi tube and with DOSY-ufCOSY with sigma $=3 \mathrm{~mm}$ ). Overall, in this case the use of spatial encoding and spatial apodisation helps to reduce the systematic error due to gradient non uniformity. 

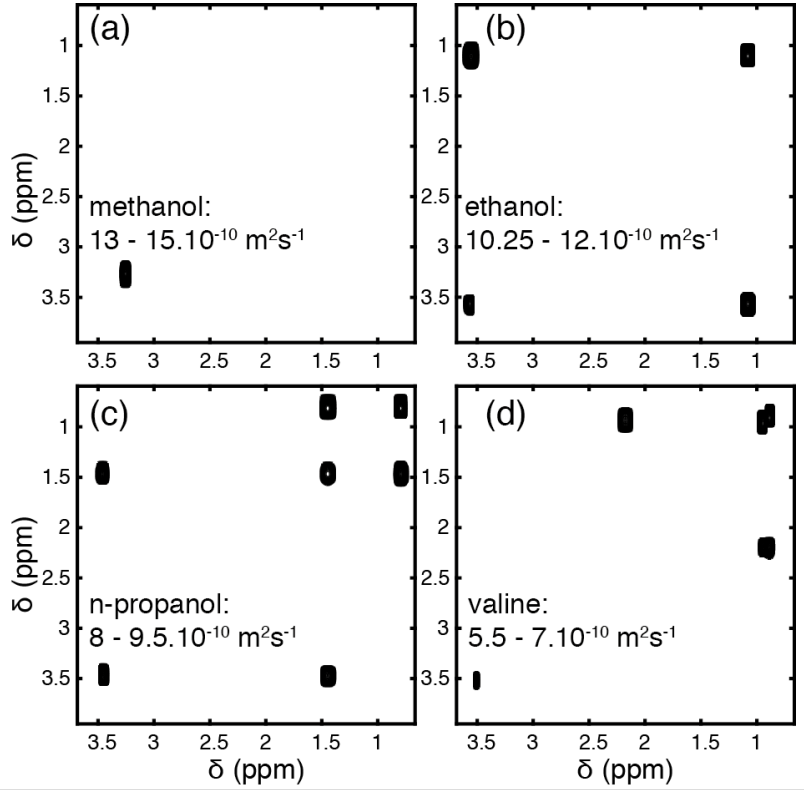

Figure 4: 3D DOSY-ufCOSY of a model mixture of three short chain alcohols and an amino acid. (a)-(d) slices extracted from the 3D spectra. The name of the compound and the selected range of $D$ in shown on each figure. spatial encoding and diffusion steps are separated (it would be needed in an alternative iDOSY approach with diffusion encoding of multiple-quantum coherences). However, it is still useful to avoid interferences between diffusion and coherenceselection gradients. For the DQS-iDOSY case, as in the conventional experiment, the choice of $\mathrm{T}$ delay is more complicated than for the COSY. It has to be long enough to let signal decrease due to diffusion and be in the good range to build up anti-phase terms which will lead to double coherences.

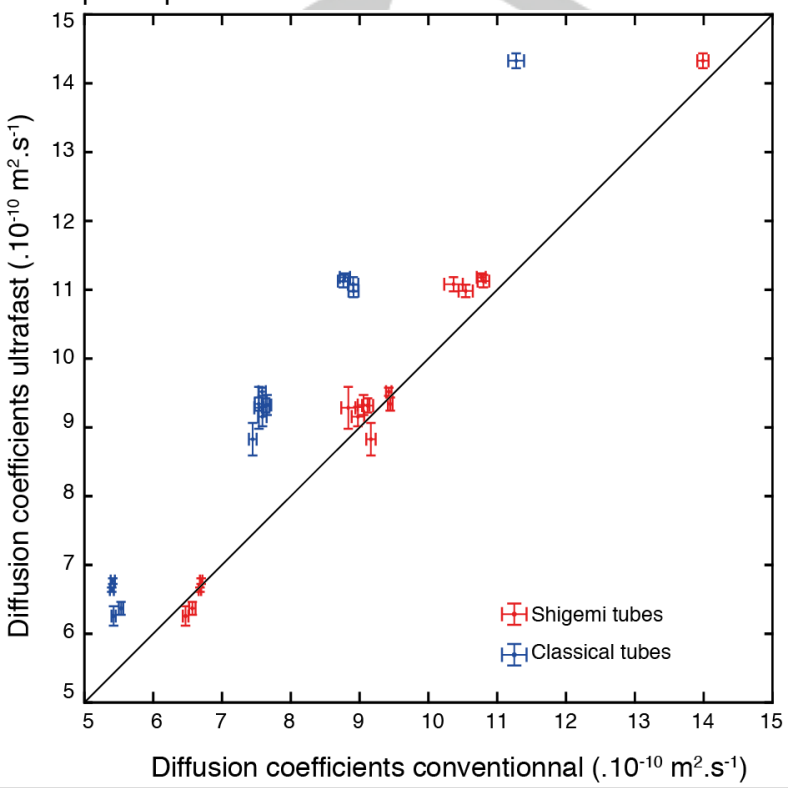

Figure 5: Comparison signal by signal between diffusion coefficients calculated from 3D DOSY-ufCOSY with 3D conventional DOSY-COSY in a classic NMR tube (blue) and in shigemi tube set with $L=10 \mathrm{~mm}$ (red). The diagonal line is shown as a guide to the eye..

Unfortunately, because of the poor sensitivity of the method (due to decrease of the signal during diffusion delay and less efficient excitation of double quanta coherences), no spectra could be recorded on the $100 \mathrm{mM}$ test sample. A more concentrated sample of ethanol and propanol was prepared (1 M). Figure 9a) shows the spectrum obtained with the weakest gradient for the ufDQS-iDOSY sequence on the new concentrated sample. On Figure $9 \mathrm{~b}-\mathrm{c}$ ) are plotted the spectra of the two compounds separated thanks their diffusion coefficient. A similar separation is obtained with the DOSY-ufDQS approach, although again with reduced sensitivity due to the additional stimulated echo.

\section{uf DQS-DOSY}

Another interesting ${ }^{1} \mathrm{H}$ homonuclear $2 \mathrm{D}$ experiment for mixture analysis is Double Quantum Spectroscopy (DQS). It has been shown to be useful for the analysis of complex samples such as biofluids ${ }^{[19,20]}$ as it yields spectra that are less crowded than COSY ones.

As in the case of COSY, two ways of writing the pulse sequence for 3D DQS-DOSY were used: DOSY-DQS and DQSiDOSY (the pulse sequences are shown in Figure 8). In this case a triple-axis gradient probe is not mandatory since the 


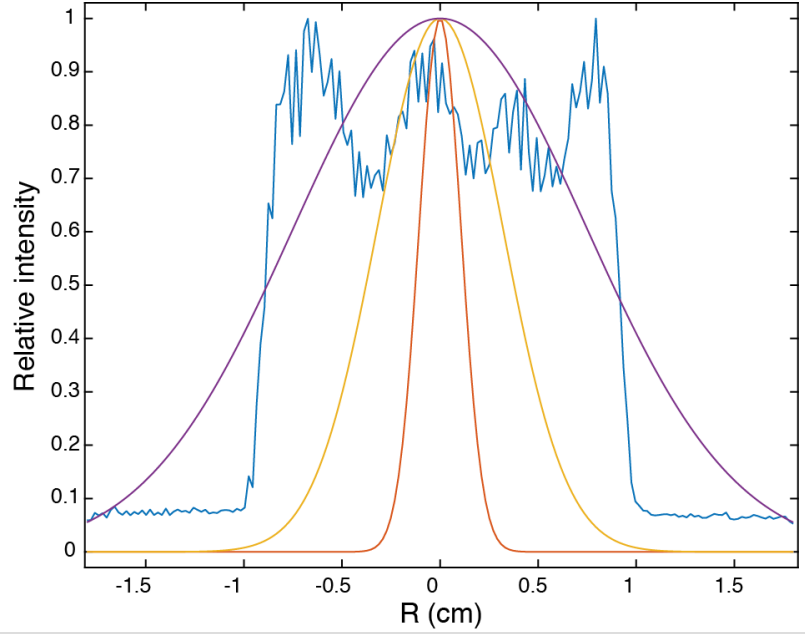

Figure 6: Spatial projection of the 2D spatial-spectral data set resulting from the first increment of the DOSY-ufCOSY experiment. The processing of UF NMR data involved multiplication by a window function in the spatial domain Three Gaussian windows are shown here, for three values of the width: $\sigma=1$ (red), 3 (yellow) and 7 (purple) $\mathrm{mm}$

Overall, while the principle of accelerating 3D DOSY with a spatial encoding of the chemical shift is also validated in the DQS case, sensitivity limitations turn out to be more significant in this case.

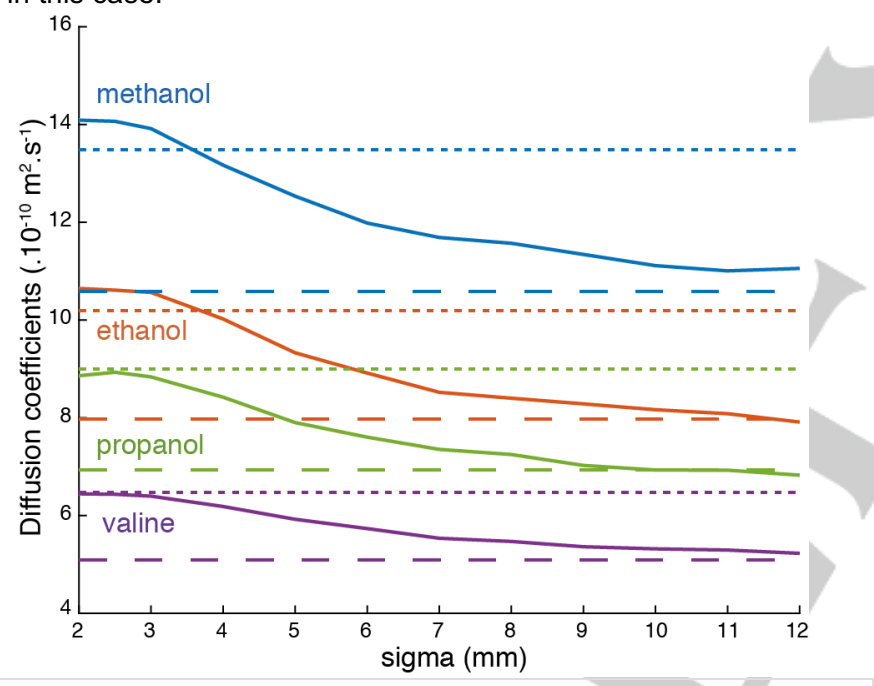

Figure 7: Estimated diffusion coefficients as a function of the spatia apodisation width (sigma) used for the processing of 3D DOSY-ufCOSY data, for a model mixture of short-chain alcohols and an amino acid.. The diffusion coefficients obtained from conventional DOSY with either a conventional tube (dashed lines) or a Shigemi tube set to $4 \mathrm{~mm}$ (dots) are displayed for comparison

\section{Spatial encoding of diffusion of chemical shifts?}

3D DOSY can be accelerated by spatial encoding of either the diffusion or the indirect spectral dimension. While the latter is described here, the former was recently reported in Ref. ${ }^{[22]}$. (in particular, a figure analogous to Fig. 4, using SPEN COSY DOSY instead of ufCOSY-DOSY, may be found in Fig. 2 of Ref. 22). Both methods provide a decrease of the experiment duration by more than one order of magnitude. However, the duration and results for these two approaches are not identical.
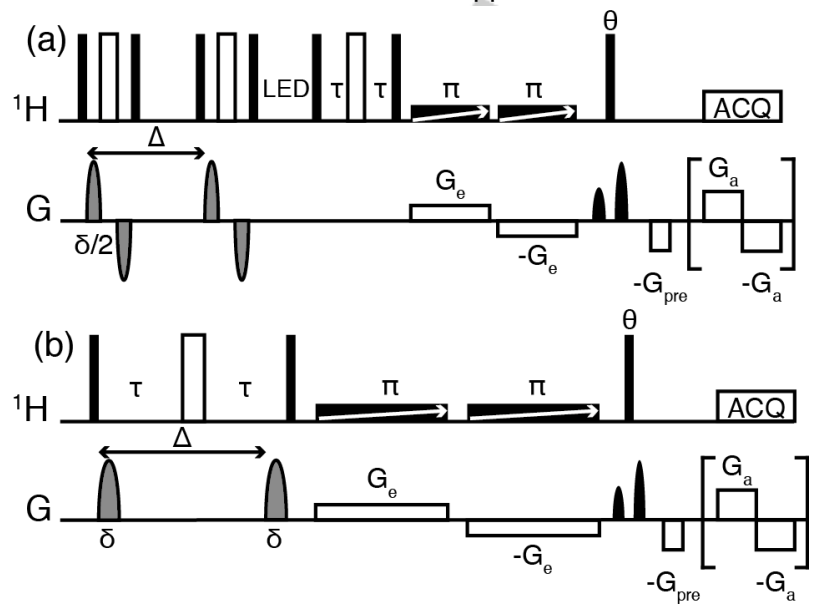

Figure 8: Pulse sequences for (a) DOSY-ufDQS and (b) ufDQS-iDOSY

The first noticeable difference is the experiment duration. When the chemical-shift information is encoded spatially, the typical number of scans required for the complete experiment is of 16 , corresponding to one scan per gradient value. When the diffusion information is encoded spatially, the typical number of scans is instead 64 or more. As a result, encoding chemical shifts spatially is 4 to 8 times shorter than encoding diffusion coefficients. Note however that this comparison assumes that magnetic-field gradients are use to perform CTP selection in a single scan. For the ufCOSY-iDOSY approach, this requires the use of triple-axis gradients. If phase cycling were used instead, the time advantage of spatially encoding chemical shifts would be lost.

One other difference between the two methods lies in the dimension (either the diffusion dimension or the indirect spectral dimension) for which resolution and accuracy are impacted by spatial encoding. With SPEN DOSY, both the trueness and precision of the diffusion information are impacted in the present implementation of the method. ${ }^{[22]}$ For ufCOSY-iDOSY, the resolution and spectral width of the indirect spectral dimension are restricted, in a well-documented way. ${ }^{[27,28]}$

The two experiments also have their spectral resolution in the direct dimension limited by the maximum acquisition time that can be achieved with classic high-resolution NMR hardware. In conventional experiment the only limit is the relaxation and the size of data, whereas in spatially encoded experiments the hardware is a limitation as the probe and the gradient amplifier cannot sustain the acquisition conditions for more than $100 \mathrm{~ms}$ usually, hence the loss in resolution in the temporal dimension compared to conventional experiments. Since the acquisition gradient intensity is lower in the SPEN DOSY COSY experiments than in the ufCOSY-iDOSY one, the former can include longer acquisition periods, leading to better resolution in the direct spectral dimension.

Spatial encoding of either the diffusion or the chemical-shift dimension also comes with a sensitivity penalty. In both cases, the signal-to-noise ratio per square root of total experiment time is predicted to decrease by a factor of $\sqrt{M}$, where $M$ is the 
number of parallelised increment. Since $M$ is larger for the ufCOSY-DOSY experiment than for the ufDOSY-COSY one, the sensitivity penalty is expected to be larger for the former. A more detailed comparison of the two approaches, included sensitivity aspects, is the topic of current investigations.
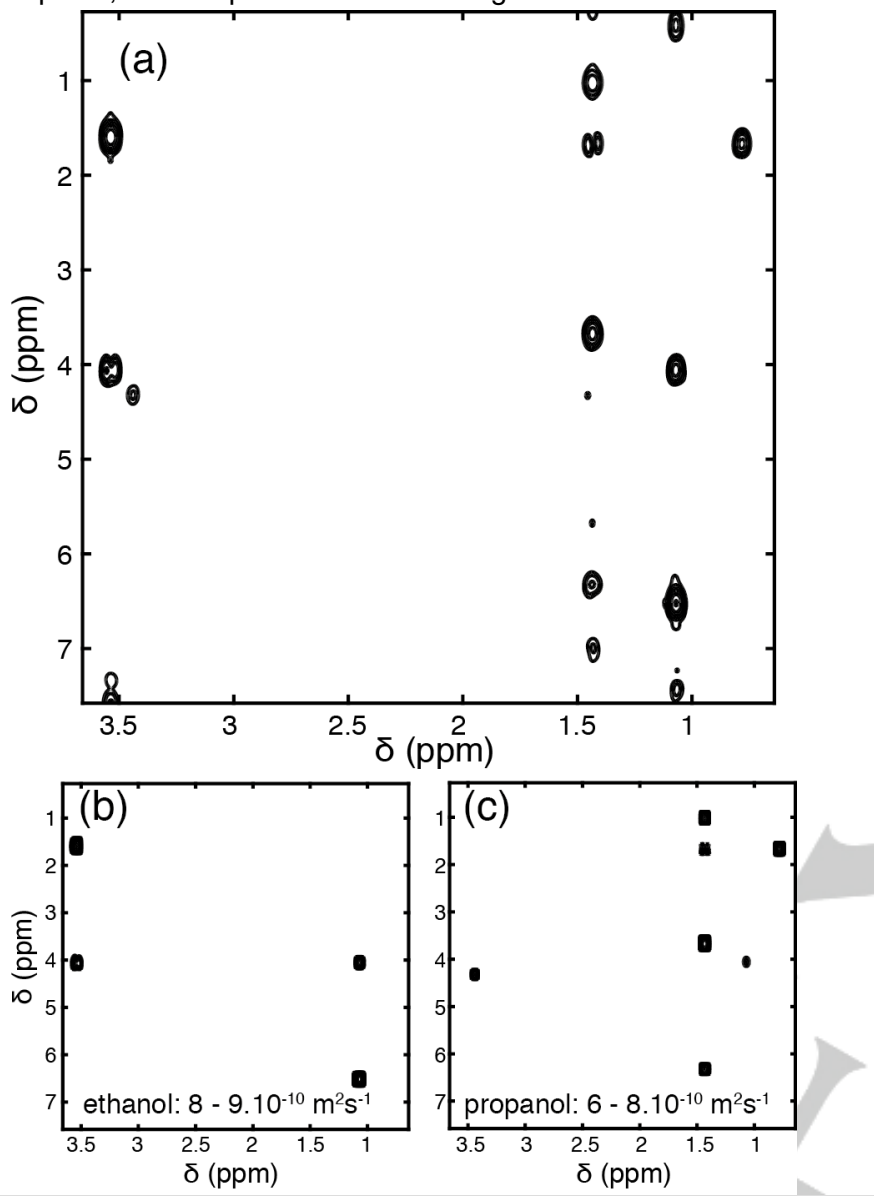

Figure 9: 3D ufDQS-iDOSY on a concentrated mixture of alcohols sample. (a) ufDQS spectrum, (b)-(c) slice extracted from 3D spectra. The selected range of $D$ in shown on each figure.

\section{Conclusions}

In summary, a fast method for 3D DOSY NMR analysis is reported here, combining the time-efficiency of ultrafast 2D NMR and the separation power of DOSY. For COSY DOSY, the concatenation and incorporation of diffusion-weighting gradients into a UF COSY experiments have been compared and the two approaches give a good separation of spectra. While the iDOSY implementation has a sensitivity advantage, it requires a tripleaxis gradient probe. The separation of UF DQS spectra has also been demonstrated but reaches the sensitivity limit of the method. Overall, 3D DOSY with a spatial encoding of the chemical shift will be useful for the time-limited analysis of mixtures.

\section{Experimental Section}

\section{Samples}

Two model mixtures of small molecules were prepared. The first mixture is composed of L-valine $(7.2 \mathrm{mg})$, methanol $(2.4 \mu \mathrm{L})$, ethanol $(3.4 \mu \mathrm{L})$ and $\mathrm{n}$-propanol $(4.5 \mu \mathrm{L})$ solubilized in $590 \mu \mathrm{L}$ of $\mathrm{D}_{2} \mathrm{O}$. The solution was shaken until complete dissolution of valine and filtered to remove any solid particles. The concentration is near $100 \mathrm{mM}$ for each compound. The second mixture is composed of ethanol $(35 \mu \mathrm{L})$ and propanol $(45 \mu \mathrm{L})$ solubilized in $520 \mu \mathrm{L}$ of $\mathrm{D}_{2} \mathrm{O}$. The concentration is near $1 \mathrm{M}$ for each compound.

Spatially encoded experiments were carried out with standard $5 \mathrm{~mm}$ tube, while conventional DOSY experiments were carried out using a $5 \mathrm{~mm}$ Shigemi tube with a restricted sample length.

\section{Experiments}

All the experiments were carried out on a Bruker Avance III spectrometer operating at a ${ }^{1} \mathrm{H}$ Larmor frequency of $600.13 \mathrm{MHz}$ and equipped with a room-temperature $5 \mathrm{~mm} \mathrm{TXI}{ }^{1} \mathrm{H} /{ }^{13} \mathrm{C} /{ }^{15} \mathrm{~N}$ probe with triple-axis gradients. The intensity of gradients was calibrated along each axis using a Shigemi tube filled with doped water $\left(1 \% \mathrm{H}_{2} \mathrm{O}+0.1 \% \mathrm{CuSO}_{4}\right.$ in $\left.\mathrm{D}_{2} \mathrm{O}\right)$ and a restricted sample length of $10 \mathrm{~mm}$. The temperature inside the probe was calibrated with a standard methanol sample and then set to a value of $298 \mathrm{~K}$ with airflow of $535 \mathrm{~L} / \mathrm{h}$. All the experiments were done without sample rotation.

Conventional 2D spectra were recorded using a stimulated echo sequence with bipolar gradient pulses (stebpgp1s Bruker sequence with additional lock stabilisation gradients).

For 3D SPEN experiments, conventional parameters for DOSY were used: a diffusion delay of $100 \mathrm{~ms}(\Delta)$ and a duration of the encoding pulse ( $\delta$ ) of $1.5 \mathrm{~ms}$ on Z-axis and $2 \mathrm{~ms}$ on X-axis. For COSY-DOSY a the diffusion-encoding gradient values consisted of a linear ramp ranging from $0.1032 \mathrm{~T} / \mathrm{m}$ to $0.5504 \mathrm{~T} / \mathrm{m}$ with 16 increments when diffusion gradients were applied on Z-axis and from $0.0762 \mathrm{~T} / \mathrm{m}$ to $0.4062 \mathrm{~T} / \mathrm{m}$ when diffusion gradients were applied on X-axis. For DQS-DOSY the diffusion-encoding gradient values consisted of a linear ramp ranging from $0.0688 \mathrm{~T} / \mathrm{m}$ to $0.5504 \mathrm{~T} / \mathrm{m}$ with 8 increments when diffusion gradients were applied on Z-axis and from $0.0508 \mathrm{~T} / \mathrm{m}$ to $0.4062 \mathrm{~T} / \mathrm{m}$ when diffusion gradients were applied on $\mathrm{X}$-axis

For ufCOSY, an encoding gradient of $0.01121 \mathrm{~T} / \mathrm{m}$ was used combined with a $15 \mathrm{~ms}$ chirp pulse with a $12 \mathrm{kHz}$ bandwidth. Acquisition parameters were a $0.4816 \mathrm{~T} / \mathrm{m}$ acquisition gradient strength with 128 gradient loops of 180 points each and a $0.767 \mu$ s dwell time. A recycle delay (D1) of $10 \mathrm{~s}, 2$ dummy scans and 1 scans per gradient increment were used, resulting in an experimental time of $2 \mathrm{~min} 55 \mathrm{~s}$.

For ufDQS, an encoding gradient of $0.00516 \mathrm{~T} / \mathrm{m}$ was used combined with a $15 \mathrm{~ms}$ chirp pulse with a $12 \mathrm{kHz}$ bandwith. Acquisition parameters were a $0.4816 \mathrm{~T} / \mathrm{m}$ acquisition gradient strength with 128 gradient loops of 180 points each and a $0.767 \mu$ s dwell time. A recycle delay (D1) of $10 \mathrm{~s}$, 2 dummy scans and 4 scans per gradient increment were used, resulting in an experimental time of $5 \mathrm{~min} 49 \mathrm{~s}$.

\section{Processing}

All the data were processed using custom-written MATLAB processing programs, adapted in part from the DOSY ToolBox for the diffusion 
dimension. ${ }^{[29]}$ Data processing is done in two steps. First, 2D spectra are reconstructed for each gradient increment. Second, 3D DOSY processing is applied to the list of $2 \mathrm{D}$ spectra.

The first step corresponds to the usual processing of UF 2D NMR data set. For each gradient increment, the data are rearranged into a $2 \mathrm{D}$ matrix and odd and even echoes are separated (only odd echoes were processed here for simplicity). Along the spatial dimension, the data is inverse Fourier transformed, apodised with a Gaussian window, zerofilled and Fourier transformed. Along the spectral dimension the data is apodised with a sine window, zero-filled and Fourier transformed. Magnitude spectra are used in all cases.

The second step corresponds to the usual processing of 3D DOSY data with magnitude spectra. Regions are defined on the spectrum obtained with the weakest diffusion gradient, and the diffusion-attenuated peak volumes are fitted with the Stejskal-Tanner equation to obtain a diffusion coefficient and a standard error for each peak. A 3D display is then constructed, with the diffusion dimension obtained by placing, for each selected spectral region, a Gaussian centred at the diffusion coefficient with a width determined by the statistics of the fit ${ }^{[29]}$. Figures show slices taken from such 3D displays.

\section{Acknowledgements}

The authors thank Ludmilla Guduff for useful discussions. This research was supported by the Agence Nationale de la Recherche (ANR-16- CE29-0012).

Keywords: NMR $\cdot$ ultrafast $•$ DOSY $\cdot 3 D \cdot$ spatial encoding

[1] H. Barjat, G. A. Morris, S. Smart, A. G. Swanson, S. C. Williams, J. Magn. Reson., 1995, 108, 170-172.

[2] C. S. Johnson Jr, Prog. Nucl. Magn. Reson. Spectrosc., 1999, 34, 203-256.

[3] R. Huo, R. Wehrens, J. van Duynhoven, L. Buydens, Anal. Chim. Acta. 2003, 490, 231-251.

[4] D. Wu, A. Chen, C. S. Johnson Jr, J. Magn. Reson. Ser. A, 1996, 121, 8891.

[5] N. Birlirakis, E. Guittet, J. Am. Chem. Soc., 1996, 118, 13083-13084.
[6] C. Dalvit, J. M. Bohlen, NMR Biomed., 1997, 10, 285-291.

[7] M. Nilsson, A. M. Gil, I. Delgadillo, G. A. Morris, Chem. Commun., 2005 1737-1739.

[8] L. H. Lucas, W. H. Otto, C. K. Larive, J. Magn. Reson., 2002, 156, 138-145.

[9] M. Urbańczyk, W. Koźmiński, K. Kazimierczuk, Angew. Chem., 2014, 126 6582-6585.

[10] S. Viel, S. Caldarelli, Chem. Commun., 2008, 2013-2015.

[11] O. Millet, M. Pons, J. Magn. Reson., 1998, 131, 166-169.

[12] L. Frydman, A. Lupulescu, T. Scherf, J. Am. Chem. Soc., 2003, 125 9204-9217.

[13] L. Frydman, T. Scherf, A. Lupulescu, Proc. Natl. Acad. Sci., 2002, 99 15858-15862.

[14] P. Pelupessy, J. Am. Chem. Soc., 2003, 125, 12345-12350.

[15] N. M. Loening, J. Keeler, G. A. Morris, J. Magn. Reson., 2001, 153, 103112.

[16] M. J. Thrippleton, N. M. Loening, J. Keeler, Magn. Reson. Chem., 2003, 41, 441-447.

[17] C. Wu, M. Zhao, S. Cai, Y. Lin, Z. Chen, J. Magn. Reson., 2010, 204, $82-$ 90

[18] P. Giraudeau, S. Akoka, J. Magn. Reson., 2007, 186, 352-357.

[19] A. Le Guennec, P. Giraudeau, S. Caldarelli, J.-N. Dumez, Chem. Commun., 2015, 51, 354-357.

[20] L. Rouger, B. Gouilleux, M. Pourchet-Gellez, J.-N. Dumez, P. Giraudeau, Analyst, 2016, 141, 1686-1692.

[21] Y. Shrot, L. Frydman, J. Magn. Reson., 2008, 195, 226-231.

[22] L. Guduff, I. Kuprov, C. van Heijenoort, J.-N. Dumez, Chem. Commun., 2017, 53, 701-704.

[23] S. Ahola, V. V. Zhivonitko, O. Mankinen, G. Zhang, A. M. Kantola, H.-Y. Chen, C. Hilty, I. V. Koptyug, V.-V. Telkki, Nat. Commun., 2015, 6.

[24] M. D. Pelta, G. A. Morris, M. J. Stchedroff, S. J. Hammond, Magn. Reson. Chem., 2002, 40, S147-S152.

[25] A. Bax, R. Freeman, J. Magn. Reson., 1981, 44, 542-561.

[26] M. A. Connell, P. J. Bowyer, P. A. Bone, A. L. Davis, A. G. Swanson, M. Nilsson, G. A. Morris, J. Magn. Reson., 2009, 198, 121-131.

[27] P. Giraudeau, L. Frydman, Annu. Rev. Anal. Chem., 2014, 7, 129-161.

[28] J.-N. Dumez, Prog. Nucl. Magn. Reson. Spectrosc., 2018, in press.

[29] M. Nilsson, J. Magn. Reson., 2009, 200, 296-302.

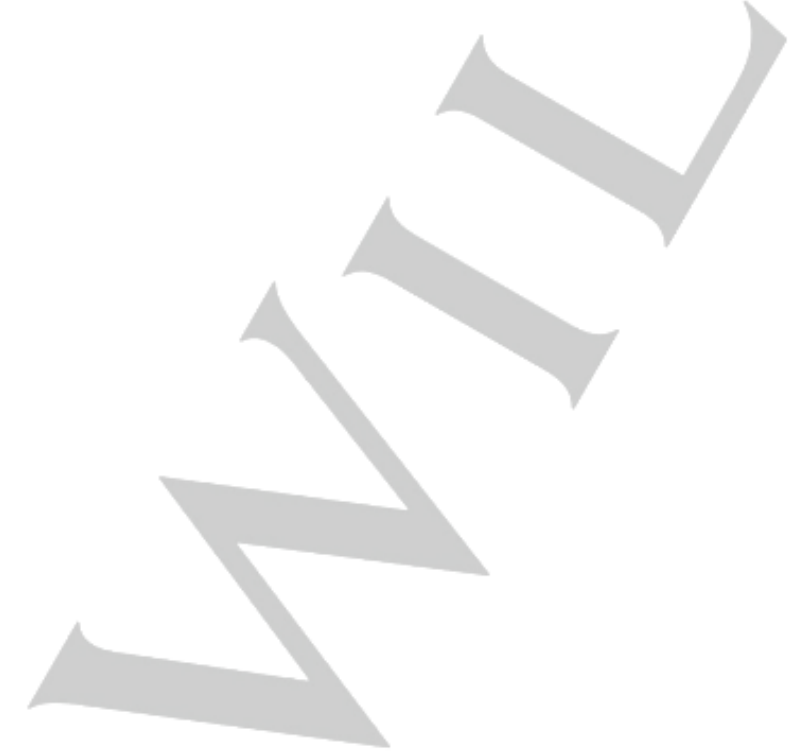


Entry for the Table of Contents (Please choose one layout)

Layout 1:

\section{ARTICLE}

Spatial encoding of chemical shift has been used to accelerate 3D COSYDOSY

Layout 2:

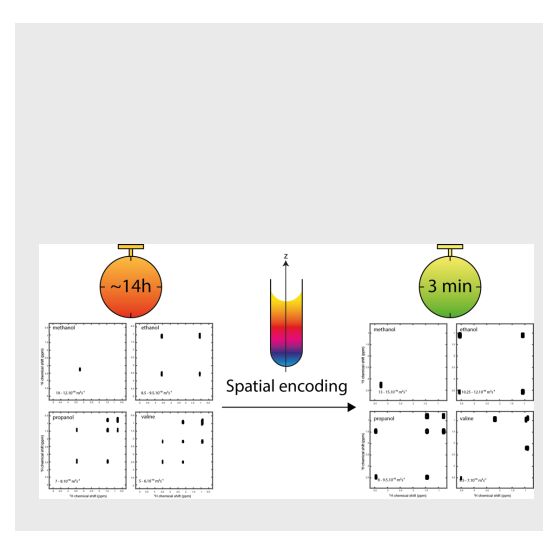

Corentin Jacquemmoz, Jean-Nicolas Dumez*

Page No. - Page No.

Title

Author(s), Corresponding Author(s)*

Page No. - Page No.

Title 